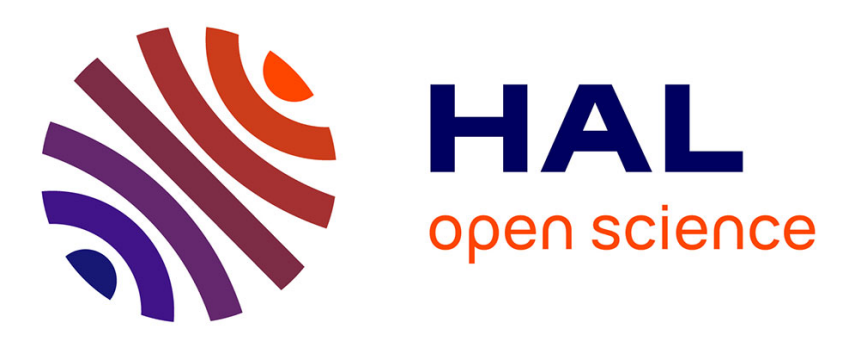

\title{
The Vertical Optic Flow: An Additional Cue for Stabilizing Beerotor Robot's Flight Without IMU
}

Fabien Expert, Franck Ruffier

\section{To cite this version:}

Fabien Expert, Franck Ruffier. The Vertical Optic Flow: An Additional Cue for Stabilizing Beerotor Robot's Flight Without IMU. Wilson, P. Stuart; Verschure, F.M.J. Paul ; Mura, Anna; Prescott, J. Tony. Biomimetic and Biohybrid Systems: 4th International Conference, Living Machines 2015, Barcelona, Spain, July 28 - 31, 2015, Proceedings, Springer, pp.187-198, 2015, 978-3-319-22979-9. 10.1007/978-3-319-22979-9_19 . hal-01315451

\section{HAL Id: hal-01315451 https://hal.science/hal-01315451}

Submitted on 13 May 2016

HAL is a multi-disciplinary open access archive for the deposit and dissemination of scientific research documents, whether they are published or not. The documents may come from teaching and research institutions in France or abroad, or from public or private research centers.
L'archive ouverte pluridisciplinaire HAL, est destinée au dépôt et à la diffusion de documents scientifiques de niveau recherche, publiés ou non, émanant des établissements d'enseignement et de recherche français ou étrangers, des laboratoires publics ou privés. 


\title{
The vertical Optic Flow : an additional cue for stabilizing Beerotor robot's flight without IMU
}

\author{
Fabien Expert and Franck Ruffier \\ Aix Marseille University, CNRS, ISM UMR7287, \\ Biorobotics Research Group www . biorobotics.eu, \\ F-13288, Marseille, France \\ franck.ruffier@univ-amu.fr \\ www.ism.univ-amu.fr
}

\begin{abstract}
Bio-inspired guidance principles involving no reference frame are presented here and were implemented in a rotorcraft called Beerotor, which was equipped with a minimalistic panoramic optic flow sensor and no accelerometer, no inertial measurement unit (IMU) [9], as in flying insects (Dipterian only uses rotation rates). In the present paper, the vertical optic flow was used as an additional cue whereas the previously published Beerotor II's visuo-motor system only used translational optic flow cues [9]. To test these guidance principles, we built a tethered tandem rotorcraft called Beerotor $(80 \mathrm{~g})$, which flies along a high-roofed tunnel. The aerial robot adjusts its pitch and hence its speed, hugs the ground and lands safely without any need for an inertial reference frame. The rotorcraft's altitude and forward speed are adjusted via several optic flow feedback loops piloting respectively the lift and the pitch angle on the basis of the common-mode and differential rotor speeds, respectively as well as an active system of reorientation of a quasi-panoramic eye which constantly realigns its gaze, keeping it parallel to the nearest surface followed. Safe automatic terrain following and landing were obtained with the active eye-reorientation system over rugged terrain, without any need for an inertial reference frame.
\end{abstract}

Keywords: Panoramic optic-flow, optic flow of expansion, no referenced states, bio-inspired autopilot

\section{Introduction}

Miniature insect-scale robots [15], just like Micro Aerial Vehicles (MAVs), have to be able to make their way autonomously through cluttered, partially moving environments, e.g. foliage moving with the wind, and cope with unpredictable events, e.g. vehicle or human movements. These challenging tasks may call for novel sensors and novel control methods that differ from those used in conventional approaches, where all the states of the aerial robot are either measured or estimated in the inertial reference frame [16,23].

Ethological findings have shown that complex navigation tasks such as terrain following and speed control are performed by flying insects on the basis of optic 
flow (OF) cues by means of their tiny compound eyes have a very poor spatial resolution in comparison with modern high resolution cameras. In particular, recent studies on insects have shown that the ventral $[1,2,18]$ and dorsal [19] optic flows (OFs) play an important role in altitude control.

Several authors inspired by studies on honeybee landing [24] recently started to use the optic flow as a means of landing automatically $[6,22,27,14,11,17,13$, $8,7]$.

In all robotic studies involving the use of OF, the inputs used by the autopilots of rotary-winged robots were always referred to the inertial frame provided by either an IMU $[12,13,26]$, a barometric altimeter [12] or an external actuator placed on a tether $[20,22,21,8]$. In some studies, fixed-wing robots did not have to use any inertial frame of reference $[3,10,28,4,5]$ because fixed-wing robots are naturally more stable than rotorcraft. The use of a rate gyro in an inner loop is compulsory to stabilize the roll and pitch flight dynamics of most rotary-wings based robots.

In recent studies conducted on Beerotor at our laboratory [9], the rotorcraft's altitude and forward speed are adjusted via several feedback loops based on ventral and drosal translational optic flow piloting:

- the lift and the pitch angle on the basis of the common-mode and differential rotor speeds, respectively

- an active system of reorientation based on a quasi-panoramic eye which constantly realigns its gaze, keeping it parallel to the nearest surface followed.

Safe automatic terrain following and landing were obtained with the active eye-reorientation system over rugged, changing terrain, without any need for an inertial reference frame [9].

In the present paper, we aim at incorporating the use of the vertical optic flow into our Beerotor aerial robot. Indeed, during experiments on Drosophila freely flying in a 3D virtual reality environment, Straw et al. have shown that a ventral expansion avoidance reflex is used by insects to control their altitude. The flies generated an increasing climb rate when flying over an expanding stimulus [25]. We suggest here a embodied model - a real aerial robot - where such reflex does not conflict with a ventral optic flow regulator and both could be used together with the expansion avoidance reflex only triggered by a strong stimulus overriding all others control signals and behaviors. In our case, as we are flying in translation in a tunnel with optic flow sensors looking only downward and upward, we can not measure expansion that would only appear in front of the aircraft near the focus of expansion.

Section 2 shortly describes the mechanical and electronic design of the Beerotor robot and the $12 \mathrm{~m}$-long circular experimental set-up in which the flying robot was tested. Section 3 present the Beerotor II's main feedback loops based on the optic flow measurements performed by its quasi-panoramic eye during autonomous flights. Section 4 defines the vertical Optic Flow. Section 5 presents the feedforward control loop based on the vertical OF. 


\section{Beerotor's airframe}

To test the validity of the eye-reorientation guidance principle, the Beerotor II robot (see Figure 1) was equipped with a quasi-panoramic eye (see Figure 1c) consisting of 4 visual motion sensors, each of which comprised 6 pixels only and covered a solid angle of $23^{\circ}$. This eye was placed $7 \mathrm{~cm}$ from the robot's body to prevent the propellers from entering the visual field of the eye. The robot's miniature quasi-panoramic eye and the optic flow processing scheme measured the median optic flow based on 5 local optic flow measurements delivered by neighbouring 2-pixel Local Motion Sensors (LMSs) (see[8] for more details). The robot's eye constantly realigned itself with respect to the slope of the nearest surface (see figure 7 in [9]) by means of the presence of a stepper motor coupled with a gear-reducer giving a resolution of $0.02^{\circ} /$ steps, which pitches the orientation of the eye up or down with respect to the robot's body. The visual cues (the optic flow) used by the aerial robot therefore always refer to the slope of the nearest surface and not to the absolute vertical. This eye-reorientation guidance principle enabled to perform all the optic flow measurements in the new frame of reference associated with the robot's eye, $\left(E, x_{e}, y_{e}, z_{e}\right)$, which is defined by the local slope of the surface followed.

\section{Beerotor's main visuomotor control loops}

The Beerotor autopilot relies almost exclusively on its optic flow sensors to control its eye orientation, its forward speed and its altitude by means of three main feedback loops (see figure 2). The first feedback loop (green) controls the orientation of the eye relatively to the body and always keeps the eye parallel to the closest surface by means of an angle estimation done by Least Squares approximation performed on the optic flow measurements. In particular, the reorientation strategy makes the aerial robot fly safely in the case of highly variable environments and very steep obstacles (30 slope). The second feedback loop (red) controls the altitude of the aircraft in order to always keep constant the optic flow generated by the closest surface. By taking the maximal value between the forward ventral and dorsal optic flows, the aircraft safely follows the closest surface (either the groud or the ceiling). To do so, the optic flow is compared to the Maximum OF setpoint $\omega_{\text {setMaxOF }}$ and the error is minimized by controller which acts on the vertical lift of the aircraft and therefore its altitude. The distance to the closest surface depends on the chosen setpoint and the forward speed of the robot.

The last feedback loop (blue) controls the forward speed of the robot based on the sum of the ventral and the dorsal optic flows by means of two nested feedback loops. The difference between the sum of the optic flows and a setpoint value $\omega_{\text {setSumOF }}$ is used to optionally control the rotorcraft's airspeed measured by means of a custom-made airspeed sensor and to regulate the pitch rate of the aircraft $\dot{\theta}$ by means of the rate gyro measurements. This feedback loop coupled with a ventral or dorsal regulator automatically adapts the forward speed 

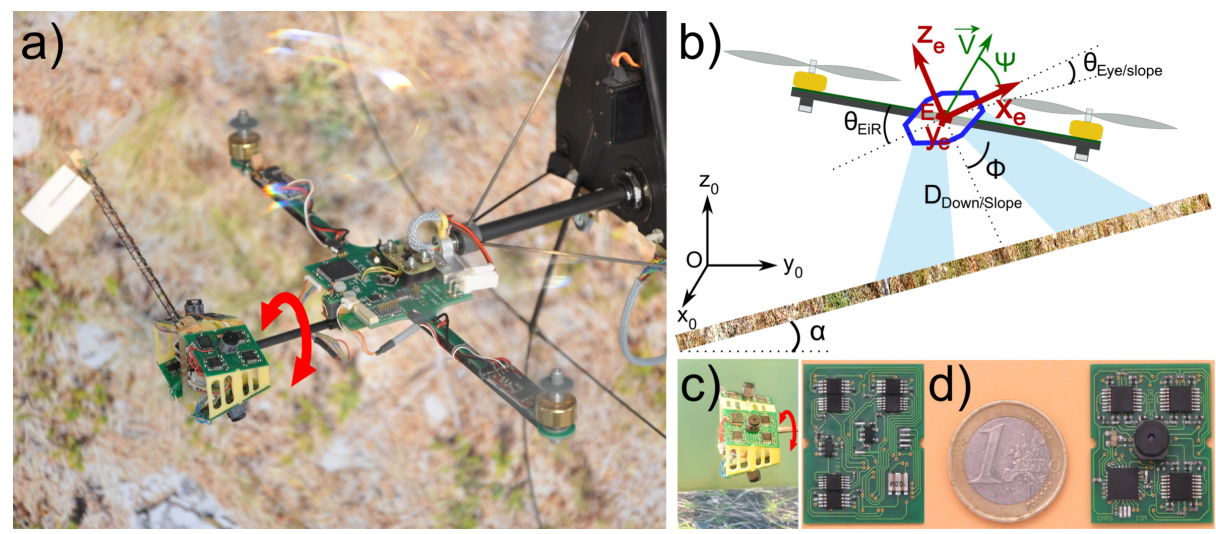

Fig. 1. a) Photograph of the $80 \mathrm{~g}$ Beerotor II robot. The Beerotor robot is equipped here with a quasi-panoramic eye decoupled from the body, which is composed of 4 visual motion sensors sampling the visual environment with a $4 \times 24^{\circ}$ FOV. b) Drawing of the Beerotor robot flying over a terrain slanting at an angle $\alpha$. The angle of the eye relative to the body $\theta_{E i R}$ is measured via a magnetic sensor and the angle $\theta_{E y e / \text { Slope }}$ between the eye's equator and the slope of the nearest surface is estimated on the basis of the optic flow (see [9] for details of the method), and the result is used to align the eye, keeping it parallel with the terrain. The aerial robot is assumed to be flying at a velocity $\vec{V}$ in the direction defined by the angle $\Psi$ ( $\Psi$ is the angle between the direction of the speed vector and the eye's equator). c) Photograph of the quasi-panoramic eye mounted on the Beerotor II robot, which constantly realigned itself with respect to the slope of the nearest surface. The orientation of the eye relative to the body can be finely adjusted via a lightweight stepper motor combined with a $\frac{1}{120}$ gear-reducer. d) Top and bottom view of the electronic board (size: $33 \times 40 \mathrm{~mm}$ ) of one Visual Motion Sensor with its lens mounted on the LSC photosensor array.

of the aircraft to the size of the tunnel where the rotorcraft is flying by reducing its speed when the tunnel is narrowing and accelerating when the tunnel is getting wider.These last two intertwined feedback loops guarantee that the Beerotor robot will always keep a safe distance from both walls while adapting its forward speed to the size of the tunnel without any measurements of distance or groundspeed. Here, we suggest that the vertical lift of the aircraft can also be controlled by a feedforward controller using the measurement of the Vertical optic flow $\omega_{V \text { VerticalOF }}^{\text {meas }}$ to reduce the altitude oscillations of the aircraft. The idea is to anticipate the changes in the ventral or dorsal optic flow during strong variations of altitude like when overflying the obstacle.

\section{Definition of the Vertical ventral or dorsal OF}

In our case, as we are flying in translation in a tunnel with optic flow sensors looking only downward and upward, we can not measure expansion that only appears in front of the aircraft near the focus of expansion. However, the differ- 


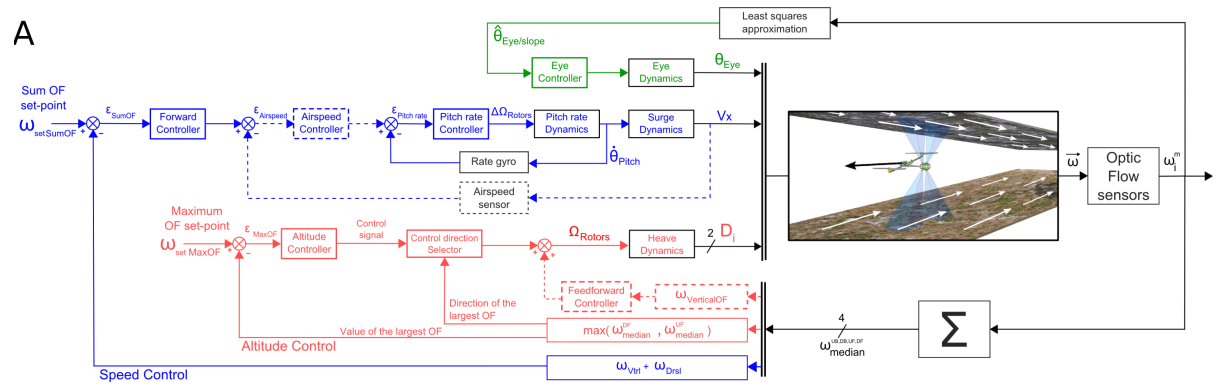

\section{B Zoom on the control system}

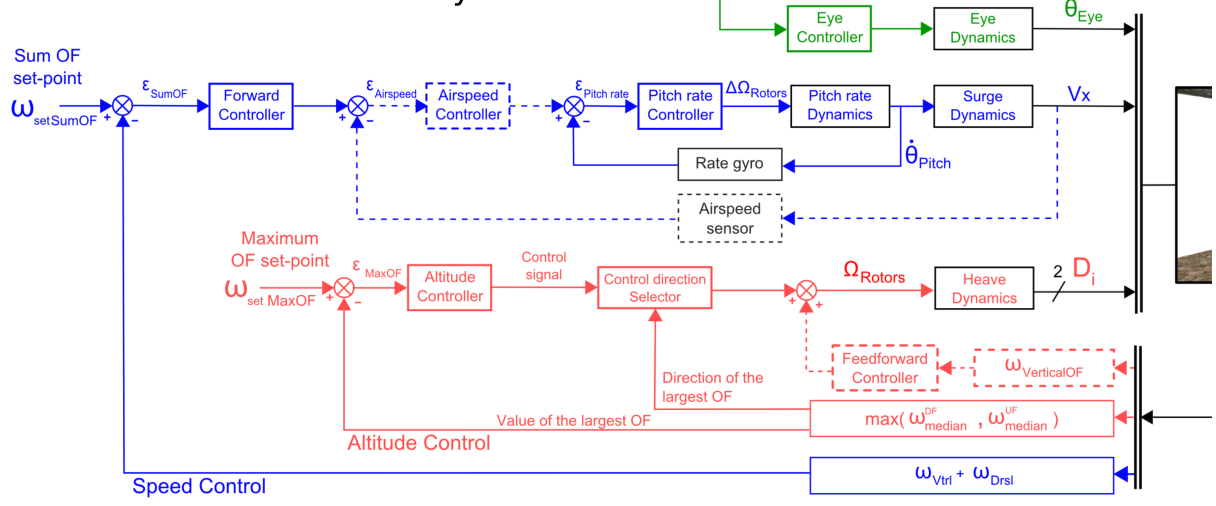

Fig. 2. (A-B) The Beerotor autopilot relies almost exclusively on its optic flow sensors to control its eye orientation, its forward speed and its altitude by means of three main feedback loops.

ence between the two ventral or dorsal optic flows $(\omega(\phi)-\omega(-\phi))$ depends on the ratio between the vertical speed $V_{z}$ and the distance to the surface $h$.

$$
\omega(\phi)=\frac{\|\vec{V}\|}{h} \cos (\phi) \cdot \cos (\psi-\phi)
$$

where $\omega$ is the angular speed, $\phi$ is a visual direction, $h=D \cdot \cos (\phi)$ is the altitude of the aircraft and $\psi$ is the angle between the eye's equator and the direction of the speed vector $\vec{V}$.

$$
\begin{gathered}
\omega(\phi)-\omega(-\phi)=\frac{\|\vec{V}\| \cdot \sin (2 \phi) \cdot \sin (\psi)}{h} \\
\omega(\phi)-\omega(-\phi)=\sin (2 \phi) \frac{V_{z}}{h}
\end{gathered}
$$

This could then be used to control the climb rate of the Beerotor robot or to detect the increasing proximity of an object and increase the distance to it. As 
the vertical speed is always smaller than the horizontal speed, this measurement will be most of the time a lot smaller than $\omega_{\text {Max OF }}$ except when the aircraft comes really close to an object.

Depending on the closest surface, the Vertical OF is computed using:

$$
\begin{aligned}
& \omega_{\text {Ventral VerticalOF }}^{\text {meas }}=\frac{1}{\sin (2 \phi)} \cdot\left(\omega_{\phi}^{\text {median }}-\omega_{-\phi}^{\text {median }}\right) \\
& \omega_{\text {Dorsal VerticalOF }}^{\text {meas }}=\frac{1}{\sin (2 \phi)} \cdot\left(\omega_{180^{\circ}+\phi}^{\text {median }}-\omega_{180^{\circ}-\phi}^{\text {median }}\right)
\end{aligned}
$$

where $\omega_{\phi}^{\text {median }}$ is the median optic flow of the local motion sensor looking in the direction $\phi$.

Coupled with a ventral optic flow regulator, the computed Vertical OF should always be close to zero as the altitude of the aircraft will always be kept constant to maintain a safe distance with the followed surface according to its forward speed. However, if the Vertical OF strongly increases or decreases, it means that the aircraft is suddenly getting closer to or increasing distance from an obstacle, respectively, information that can be used to control the robot.

\section{Feedforward control based on the Vertical OF}

To improve the performances of the Beerotor robot, we added in the altitude control loop a second feedback loop based on the measured Vertical OF $\omega_{\text {Vertical }}^{\text {meas }}$ which was used to control the altitude of the aircraft by means of a feedforward controller (see Figure 2). The output of this controller is added to the output signal of the same Altitude controller used previously. This inner loop can be said to act as a ventral or dorsal expansion avoidance reflex. Indeed, when the aircraft is flying away from obstacles, the measured Vertical OF will be low and the altitude of the aircraft will mainly be determined by the previous altitude control loop using the maximum value between the ventral and dorsal optic flows to control the thrust of the robot. On the other hand, a strong increase of the Vertical OF indicating an approaching object will cause through the feedforward controller an increase or a decrease in the vertical lift keeping the aircraft away from danger. In the same way, an important decrease of the Vertical OF will lead to a reduction or an increase of the mean speed of the propellers $\Omega_{\text {Rotors }}$.

Figure 3 shows the Beerotor robot's performance while automatically following the terrain for several initial conditions and values of the Maximum OF setpoint $\omega_{\text {setMaxOF }}$ during ten consecutive turns with an optic flow setpoint of the speed control loop $\omega_{\text {setSumOF }}=250^{\circ} / \mathrm{s}$. Regardless of the value of the Max OF setpoint, the autopilot incorporating a feedforward controller based on the Vertical OF avoided the relief. In the bottom part is represented the value of the Vertical OF which was most of the time around $0^{\circ} / \mathrm{s}$. In particular, when following the ceiling at a constant altitude, the Vertical OF did not significantly differ from $0^{\circ} / \mathrm{s}$ having no influence on the behavior of the aircraft. On the other 

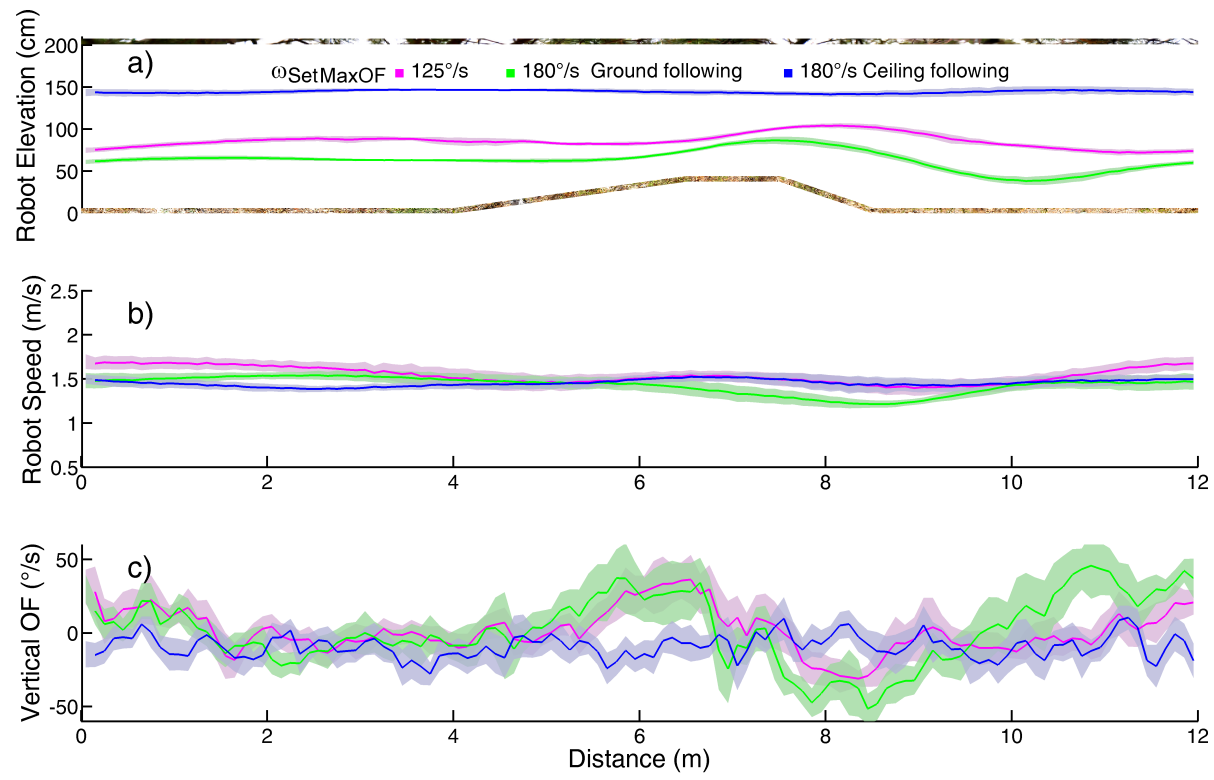

Fig. 3. Automatic ground-hugging under optic flow regulation with a feedforward controller based on the Vertical optic flow and acting on the Vertical lift. Each curve shows the mean altitude, speed and Vertical optic flow of the aircraft with the corresponding standard dispersions during 10 consecutive turns of the aircraft on its environment with an optic flow setpoint of the speed control loop $\omega_{\text {setSumOF }}=250^{\circ} / \mathrm{s}$. a) Altitude of the aircraft for three different conditions: following the closest surface with $\omega_{\text {setMaxOF }}=125^{\circ} / \mathrm{s}$ (magenta), following respectively the ground (green) and the ceiling (blue) with $\omega_{\text {setMaxOF }}=180^{\circ} / \mathrm{s}$. As expected, the aircraft perfectly avoided the slanted relief by means of the altitude control loop. When we increased the Maximum optic flow setpoint, the aircraft immediatly came closer to the closest surface in order to reach the setpoint value. By means of the feedforward controller on the Vertical OF, the oscillation after the obstacle was reduced. b) Forward speed of the rotorcraft in the three conditions. The steady-state forward speed was the same in the three experiments as the Sum OF setpoint was the same. c) Vertical OF of the aircraft. When following the ceiling, $\omega_{V \text { erticalOF }}^{\text {meas }}$ was always close to $0^{\circ} / \mathrm{s}$ whereas it increased during the ascending ramp and decreased during the descending ramp of the relief while the aircraft followed the ground.

hand, when following the ground with the slanted ground profile and in particular when the Max OF setpoint increased (green curve), the robot flew closer to the objects leading to variations of the Vertical OF that helped the rotorcraft to avoid the obstacle. In particular, we noticed that the Vertical OF decreased in relation to the changes in the floor profile when the aircraft was flying down to restore its ventral optic flow leading to an increase in the thrust of the propellers by means of the feedforward controller. This is particularly interesting as 


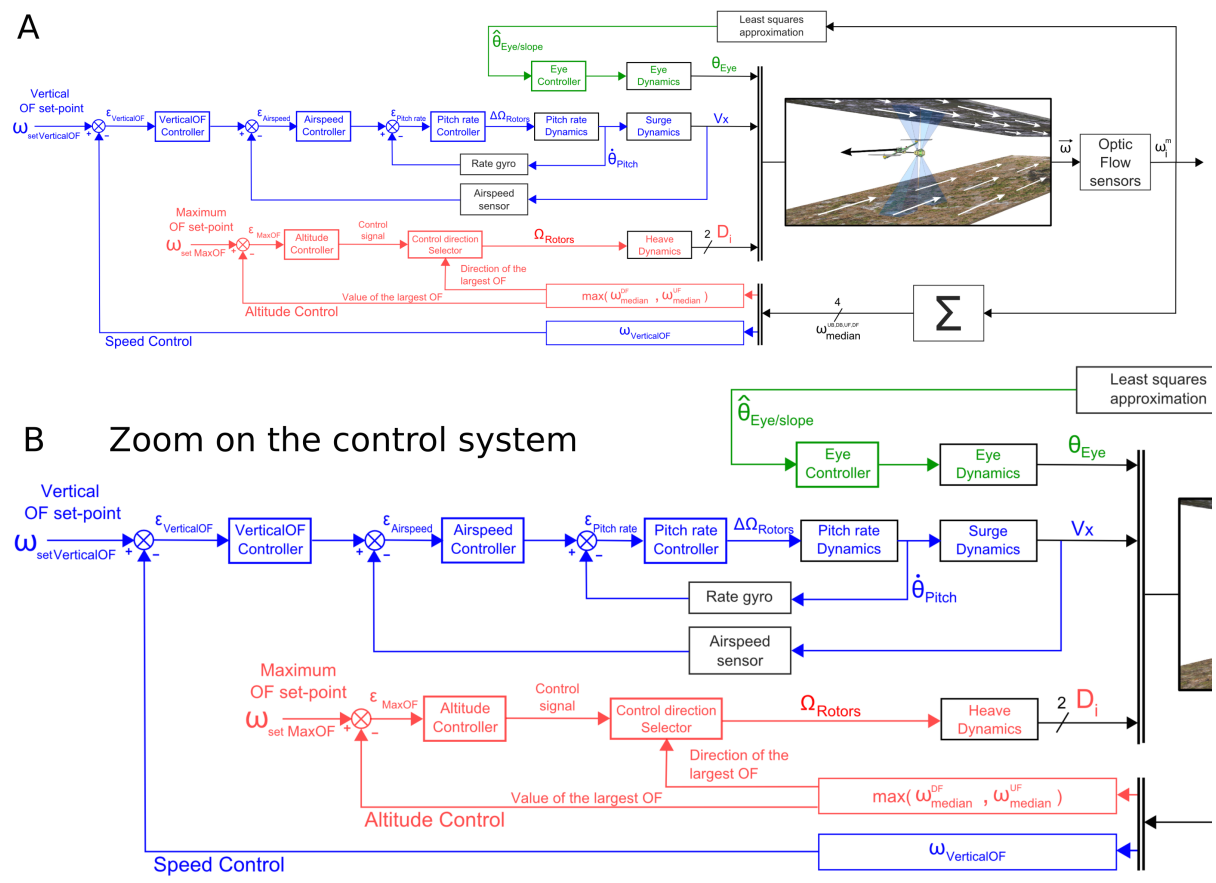

Fig. 4. (A-B) Beerotor autopilot with a speed control loop based on the Vertical optic flow for landing.

it limited the amplitude of the aircraft's altitude undershoot after the changes in the ground profile.

Although not strongly affecting the behavior of the aircraft with our experimental setup, such strategy would allow freely flying aircraft to more robustly avoid obstacles and therefore navigate collision-free in an unknown environment.

\section{Regulation of the Vertical OF during landing}

As we have seen in section 4, the Vertical optic flow is proportional to the ratio between the vertical speed $V_{z}$ and the distance $h$ to the surrounding objects. By means of the altitude control loop regulating the ventral or dorsal optic flow, the distance $h$ to the objects is theoretically always kept constant. By coupling the altitude control loop with a feedback loop controlling the forward speed of the aircraft based on the vertical OF, we can induce an automatic landing. Indeed, by taking the difference between a positive setpoint value $\omega_{\text {setVerticalOF }}$ and the measured Vertical OF and use it to drive a lead phase regulator controlling the robot forward speed by means of the two already presented nested feedback loops acting on the airspeed and the pitch rate, the aircraft will automatically reduce its forward speed in order to generate a non null vertical speed by means 

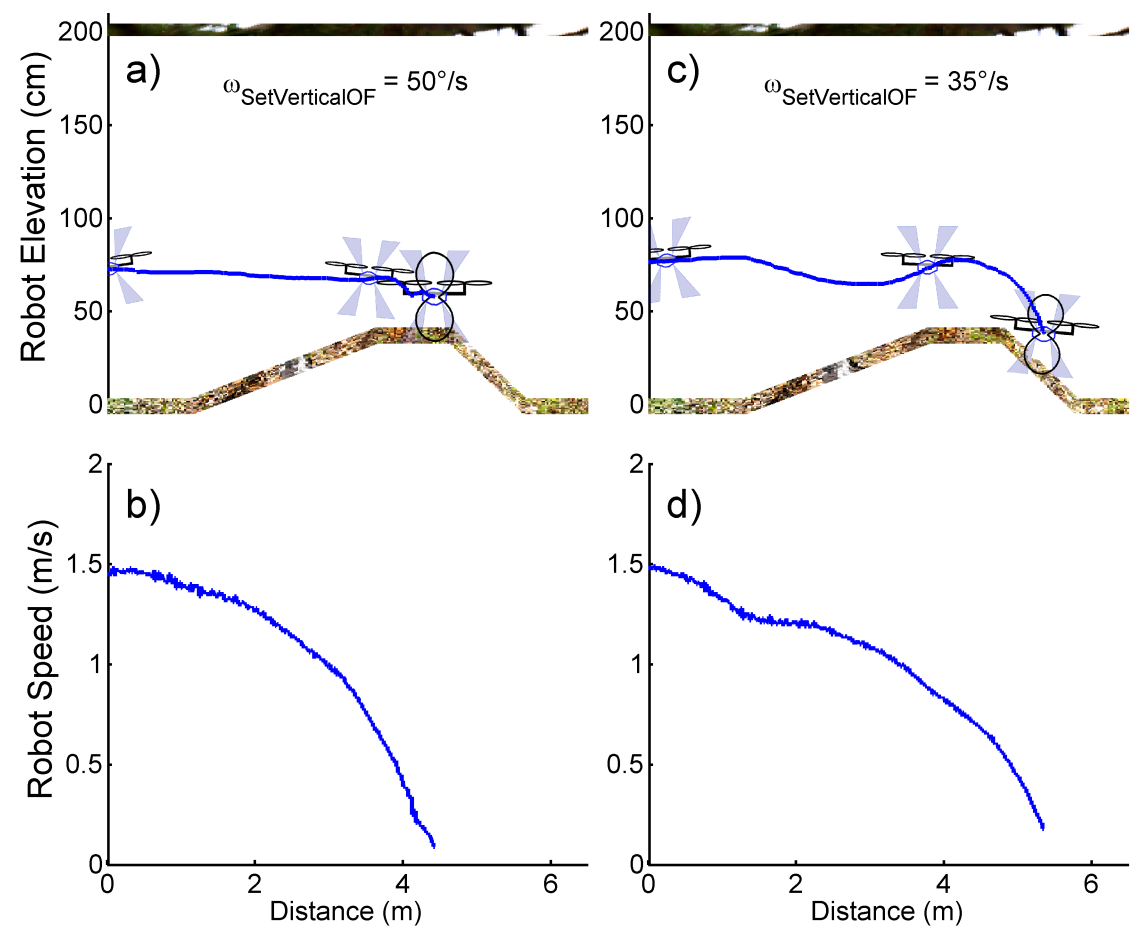

Fig. 5. Automatic landing of the aircraft on the ground achieved by controlling the forward speed feedback loop with the Vertical optic flow. a-c) Altitude of the Beerotor robot during automatic landing with $\omega_{\text {setMaxOF }}=150^{\circ} / \mathrm{s}$ and two different values of the Vertical OF setpoint $\omega_{\text {setVerticalOF }}=50^{\circ} / \mathrm{s}$ and $\omega_{\text {setVerticalOF }}=35^{\circ} / \mathrm{s}$. In both cases, the aircraft successfully landed and the duration of the landing increased when the Vertical OF setpoint $\omega_{\text {setVerticaloF }}$ decreased. b-d) To reach the setpoint value $\omega_{\text {setVerticalOF }}$, the rotorcraft reduced its forward speed leading to a loss of altitude induced by the feedback loop regulating the ventral optic flow and hence a smooth landing with almost no speed at touchdown.

of the altitude control loop. Such strategy leads to a smooth landing with almost no speed at touchdown where the duration of the landing depends on the chosen setpoint $\omega_{\text {setVerticalOF }}$. The proposed autopilot is presented in figure 4 with the altitude control loop (in red) using the maximum value between the ventral or the dorsal optic flow compared with a setpoint value to act on the vertical lift of the aircraft and therefore its altitude. Contrary to the previous experiments, the speed control loop (in blue) is here based on the difference between a fixed setpoint and the measured Vertical OF $\omega_{\text {VerticalOF }}^{\text {meas }}$ which is used to control the forward speed of the Beerotor robot. The Vertical OF controller is in that case 
a simple phase lead controller increasing the damping of the system and hence its stability.

Figure 5 shows the automatic landing of the aircraft obtained when the forward speed of the aircraft was controlled by the vertical OF for two different values of the Vertical OF setpoint: $50^{\circ} / \mathrm{s}$ and $35^{\circ} / \mathrm{s}$. In any case, the aircraft immediately decreased its forward speed and therefore came nearer to the ground as the altitude control loop decreased the vertical lift to keep its ventral optic flow constant. As expected, the slope of the deceleration increased with the setpoint value allowing to control the descent speed of the aircraft or the duration of the landing procedure. In conclusion, the Vertical OF can not only be used as a ventral expansion reflex but also to control the vertical speed of the aircraft leading to a smooth landing on the ground at a forward speed close to $0 \mathrm{~m} / \mathrm{s}$.

\section{Conclusion}

We suggested new visuo-motor feedback loops that exploit suitably the expansion components in the vertical $\mathrm{OF}$ in addition to the translational ventral and dorsal OF. Beerotor is able to fly in a steeply sloping environment without an accelerometer and without any need to refer to the absolute vertical.

The main limitation of the Beerotor robot is its mechanical tether which limits its dynamics range and the number of degree of freedom compared to an aerial robot that would freely fly in 3D space.

The advantages of not using an accelerometer are that the strategies we presented here could be embedded into the lightest of robots, such as the insect-scale aerial robot weighing only a few hundred milligrams recently developed by [15].

\section{Acknowledgments}

We are very grateful to M. Boyron for his involvement in the electronic design, F. Paganucci, Y. Luparini and J. Diperi for their help with the mechanical design, J. Blanc for improving the English manuscript and A. Manecy, G. Sabiron, G. Portelli, J. Serres, N. Franceschini and S. Viollet for their fruitful comments and suggestions during this research. This work was supported partly by CNRS Institutes (Life Science; Information Science; Engineering Science and Technology), the Aix- Marseille University, the French National Research Agency -ANR(EVA project under ANR-ContInt grant number: ANR-08-CORD-007-04) and the European Commission via the CURVACE project. The CURVACE project acknowledges the financial support of the Future and Emerging Technologies (FET) programme within the Seventh Framework Programme for Research of the European Commission, under FET-Open grant number: 237940. 


\section{References}

1. Baird, E., Srinivasan, M., Zhang, S., Lamont, R., Cowling, A.: Visual control of flight speed and height in the honeybee. In: Nolfi, S., Baldassarre, G., Calabretta, R., Hallam, J., Marocco, D., Meyer, J.A., Miglino, O., Parisi, D. (eds.) From Animals to Animats 9, vol. 4095, pp. 40-51. Springer Berlin / Heidelberg (2006)

2. Barron, A., Srinivasan, M.: Visual regulation of ground speed and headwind compensation in freely flying honey bees (apis mellifera 1.). Journal of Experimental Biology 209 (5), 978-984 (2006)

3. Barrows, G., Neely, C.: Mixed-mode VLSI optic flow sensors for inflight control of a Micro Air Vehicle. In: SPIE : Critical technologies for the future of computing. vol. 4109, pp. 52-63. San Diego, USA (2000)

4. Beyeler, A., Zufferey, J.C., Floreano, D.: Optipilot: control of takeoff and landing using optic flow. In: European Micro Aerial Vehicle Conference (EMAV). pp. 1-8. Delft, Netherlands (2009)

5. Beyeler, A., Zufferey, J.C., Floreano, D.: Vision-based control of nearobstacle flight. Autonomous robots 27, 201-219 (2009)

6. Chahl, J., Srinivasan, M., Zhang, S.: Landing strategies in honeybees and applications to uninhabited airborne vehicles. The International Journal of Robotics Research 23, 101-110 (2004)

7. de Croon, G., Ho, H., de Wagter, C., van Kampen, E., Remes, B., Chu, Q.: Optic-flow based slope estimation for autonomous landing. International Journal of Micro Air Vehicles 5(4), 287-297 (2013)

8. Expert, F., Ruffier, F.: Controlling docking, altitude and speed in a circular high-roofed tunnel thanks to the optic flow. In: IEEE Int. Conf. on Robots and Systems (IROS). pp. 1125-1132. Vilamoura, Portugal (October 2012)

9. Expert, F., Ruffier, F.: Flying over uneven moving terrain based on optic-flow cues without any need for reference frames or accelerometers. Bioinspiration \& Biomimetics $10(2), 026003$ (2015)

10. Green, W., Oh, P., Barrows, G.: Flying insect inspired vision for autonomous aerial robot maneuvers in near-earth environments. In: IEEE Int. Conf. on Robotics and Automation (ICRA) (2004)

11. Herisse, B., Hamel, T., Mahony, R., Russotto, F.X.: The landing problem of a VTOL unmanned aerial vehicle on a moving platform using optical flow. In: IEEE International Conference on Intelligent Robots and Systems (IROS). pp. 77 - 89. Taipei, Taiwan (2010)

12. Hérissé, B., Hamel, T., Mahony, R., Russotto, F.X.: A terrain-following control approach for a vtol unmanned aerial vehicle using average optical flow. Autonomous robots 29 (3-4), 381-399 (2010)

13. Herisse, B., Hamel, T., Mahony, R., Russotto, F.X.: Landing a VTOL Unmanned Aerial Vehicle on a moving platform using optical flow. IEEE Transactions on Robotics 28(1), 77-89 (2012)

14. Kendoul, F., Yu, Z., Nonami, K.: Guidance and nonlinear control system for autonomous flight of minirotorcraft unmanned aerial vehicles. Journal of Field Robotics 27(3), 311-334 (2010), http:// onlinelibrary.wiley.com/doi/10.1002/rob.20327/pdf 
15. Ma, K., Chirarattananon, P., Fuller, S., Wood, R.: Controlled flight of a biologically inspired, insect-scale robot. Science 340 (6132), 603-607 (2013)

16. Mellinger, D., Michael, N., Kumar, V.: Trajectory generation and control for precise aggressive maneuvers with quadrotors. International Journal of Robotics Research 31 (5), 664-674 (2012)

17. Moore, R., Thurrowgood, S., Bland, D., Soccol, D., Srinivasan, M.: Uav altitude and attitude stabilisation using a coaxial stereo vision system. In: IEEE Int. Conf. on Robotics and Automation (ICRA). pp. 29-34. Anchorage, USA (2010)

18. Portelli, G., Ruffier, F., Franceschini, N.: Honeybees change their height to restore their optic flow. Journal of Comparative Physiology 196 (4), 307-313 (2010)

19. Portelli, G., Ruffier, F., Roubieu, F., Franceschini, N.: Honeybees' speed depends on dorsal as well as lateral, ventral and frontal optic flows. PLOS ONE 6 (5), doi:10.1371/journal.pone.0019486 (2011)

20. Ruffier, F., Franceschini, N.: Visually guided micro-aerial vehicle: automatic take off, terrain following, landing and wind reaction. In: Proceeding of IEEE Int. Conf. on Robotics and Automation (ICRA). pp. 2339-2346. New Orleans, USA (2004)

21. Ruffier, F., Franceschini, N.: Optic flow regulation in unsteady environments : A tethered mav achieves terrain following and targeted landing over a moving platform. In: Journal of Intelligent and Robotic Systems (2014)

22. Ruffier, F., Franceschini, N.: Optic flow regulation: the key to aircraft automatic guidance. Robotics and Autonomous Systems 50(4), 177 194 (2005)

23. Shen, S.and Mulgaonkar, Y., Michael, N., Kumar, V.: Visionbased state estimation for autonomous rotorcraft mavs in complex environments. In: IEEE International Conference on Robotics and Automation (ICRA). pp. 1758 - 1764. Karlsruhe, Germany (May 2013)

24. Srinivasan, M., Zhang, S., Chahl, J., Barth, E., Venkatesh, S.: How honeybees make grazing landings on flat surfaces. Biological Cybernetics 83, 171-183 (2000)

25. Straw, A., Lee, S., Dickinson, M.: Visual control of altitude in flying Drosophila. Current Biology 20(17), 1550-1556 (2010)

26. Strydom, R., Thurrowgood, S., Srinivasan, M.: Visual odometry: Autonomous uav navigation using optic flow and stereo. In: Australasian Conf. on Robotics and Automation (ACRA). Melbourne, Australia (December 2014)

27. Zufferey, J.C., Beyeler, A., Floreano, D.: Autonomous flight at low altitude using light sensors and little computational power. Journal of Micro Air Vehicles 2 (2), 107-117 (2010)

28. Zufferey, J.C., Floreano, D.: Fly-inspired visual steering of ultralight indoor aircraft. IEEE Transactions on Robotics 22(1), 137-146 (2006) 